International Journal of Pure and Applied Mathematics

Volume 86 No. 1 2013, 65-82

ISSN: 1311-8080 (printed version); ISSN: 1314-3395 (on-line version)

url: http://www.ijpam.eu

doi: http://dx.doi.org/10.12732/ijpam.v86i1.7

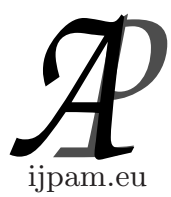

\title{
A NOTE ON TWO POINT TAYLOR EXPANSION II
}

\author{
Kazuaki Kitahara $^{1 \S}$, Takako Yamada ${ }^{2}$, Kazuki Fujiwara ${ }^{3}$ \\ ${ }^{1,3}$ School of Science and Technology \\ Kwansei Gakuin University \\ Sanda, 669-1337, JAPAN \\ ${ }^{2}$ School of Policy Studies \\ Kwansei Gakuin University \\ Sanda, 669-1337, JAPAN
}

\begin{abstract}
Let $f$ be a continuous function on $[-r, r](r>1+\sqrt{2}), \alpha$ the function $f$ restricted to the subinterval $[0, r]$ and $\beta$ the function $f$ restricted to the subinterval $[-r, 0]$. If $\alpha$ (resp. $\beta$ ) is expressed as the Taylor expansion of $\alpha$ (resp. $\beta$ ) about 1 (resp. -1 ), then we show that $f$ is expressed as the two point Taylor expansion about $-1,1$ on the interval $(-\sqrt{2}, \sqrt{2})$. Furthermore, the $k$ th order derivatives of $f$ on $(-\sqrt{2}, 0) \cup(0, \sqrt{2})$ are expressed as the termwise $k$ times differentiation of the two point Taylor expansion about $-1,1$.
\end{abstract}

AMS Subject Classification: 41A10, 41A05, 41A58

Key Words: Hermite interpolation, Taylor expansion, termwise differentiation

\section{Introduction}

As is well known, polynomial approximation has a long history and lays the foundation of approximation theory. Especially, interpolations by polynomials play an important role of polynomial approximation and have been furnishing a lot of challenging topics. Before stating the purpose of this note, we briefly review Hermite interpolation by polynomials.

Received: March 3, 2013

(C) 2013 Academic Publications, Ltd.

${ }^{\S}$ Correspondence author url: www.acadpubl.eu 
Let $I$ be an infinite subset of $\mathbf{R}$ and let $f$ be a real-valued function on $I$. For any given $(n+1)$ distinct points $X: x_{0}, \ldots, x_{n}$ in the interior of $I$ and for any sequence of positive integers $k_{0}, \ldots, k_{n}$, if $f$ is sufficiently differentiable at $x_{0}, \ldots, x_{n}$, then there exists a unique approximating polynomial $p_{f, X\left(k_{0}, \ldots, k_{n}\right)}(x)$ to $f$ which is of degree at most $m\left(=k_{0}+\cdots+k_{n}-1\right)$ and satisfies that

$$
p_{f, X\left(k_{0}, \ldots, k_{n}\right)}^{(j)}\left(x_{i}\right)=f^{(j)}\left(x_{i}\right), \quad 0 \leqq i \leqq n, 0 \leqq j \leqq k_{i}-1
$$

The points $x_{0}, \ldots, x_{n}$ and the polynomial $p_{f, X\left(k_{0}, \ldots, k_{n}\right)}$ are called nodes and the Hermite interpolating polynomial to $f$ at $x_{0}, \ldots, x_{n}$ with multiplicities $k_{0}, \ldots, k_{n}$, respectively. It is well known that for one node $X: x_{0}$ with multiplicity $n$, the Hermite interpolating polynomial $p_{f, X(n)}$ to $f$ is the Taylor polynomial of $f$ about $x_{0}$, that is,

$$
p_{f, X(n)}(x)=f\left(x_{0}\right)+\frac{f^{\prime}\left(x_{0}\right)}{1 !}\left(x-x_{0}\right)+\cdots+\frac{f^{(n-1)}\left(x_{0}\right)}{(n-1) !}\left(x-x_{0}\right)^{n-1} .
$$

Furthermore, if $f$ is infinitely differentiable at $x_{0}$ and if $f(x)=\lim _{n \rightarrow \infty} p_{f, X(n)}(x)$ for all $x \in\left(x_{0}-\rho, x_{0}+\rho\right)$ ( $\rho$ is some positive number $)$ then $f$ has the Taylor expansion of $f$ about $x_{0}$ on $\left(x_{0}-\rho, x_{0}+\rho\right)$. By this fact, we make the following definition.

Definition 1. Let $f$ be a real-valued function on a subset $I$ of the real line. If there exists a list $X$ consisting of $m$ distinct nodes $x_{0}, \ldots, x_{m-1}$ in the interior of $I$ such that $f$ is infinitely differentiable at $x_{0}, \ldots, x_{m-1}$ and

$$
\lim _{n \rightarrow \infty} p_{f, X(n, \ldots, n)}(x)=f(x) \quad \text { for all } x \in I,
$$

then it is said that $f$ has the $m$ point Taylor expansion about $x_{0}, \ldots, x_{m-1}$ on $I$. And the set of all functions to have $m$ point Taylor expansion on $I$ is denoted by $\mathcal{T}_{m}[I]$.

The notion of two point or $m$ point Taylor expansion is not new. One can see some representations of $p_{f, X(n, \ldots, n)}(x)$ in Davis[1; p.37] and the theory of $m$ point Taylor expansion in the complex plane in Walsh[6; chap.3]. López and Temme[4, 5] stated how $m$ point Taylor expansion in the complex plane can be used in deriving uniform asymptotic expansions of integrals. Furthermore, Kitahara, Chiyonobu and Tsukamoto[3] shows the following result about functions which belong to $\mathcal{T}_{2}[(-\sqrt{2}, 0) \cup(0, \sqrt{2})]$ or $\mathcal{T}_{2}[(-\sqrt{2}, \sqrt{2})]$ : 
Theorem 1. Let $f$ be a function on $\mathbf{R}$, which is expressed as

$$
f(x)=\left\{\begin{array}{ll}
\alpha(x) & x \in[0, \infty) \\
\beta(x) & x \in(-\infty, 0)
\end{array},\right.
$$

where $\alpha$ and $\beta$ are polynomials of degree at most $m$. Let $P_{\ell}, \ell \in \mathbf{N}$ be the Hermite interpolating polynomials to $f$ at $-1,1$ with multiplicities $\ell, \ell$. Then, the following assertions hold:

(1) $f$ has the two point Taylor expansion about $-1,1$ on $(-\sqrt{2}, 0) \cup(0, \sqrt{2})$, that is,

$$
\lim _{\ell \rightarrow \infty} P_{\ell}(x)=f(x), \quad \text { for all } x \in(-\sqrt{2}, 0) \cup(0, \sqrt{2}) \text {. }
$$

(2) Moreover, if $\alpha(0)=\beta(-0)$, then $f$ has the two point Taylor expansion about $-1,1$ on $(-\sqrt{2}, \sqrt{2})$, that is,

$$
\lim _{\ell \rightarrow \infty} P_{\ell}(x)=f(x), \quad \text { for all } x \in(-\sqrt{2}, \sqrt{2}) .
$$

In this note, we will show the following results of two point Taylor expansions which are related to Theorem 1.

Theorem 2. (An Extension of Theorem 1) Let $f$ be a real-valued function on $[-r, r](r>1+\sqrt{2})$, which is expressed as

$$
f(x)=\left\{\begin{array}{ll}
\alpha(x) & x \in[0, r] \\
\beta(x) & x \in[-r, 0)
\end{array},\right.
$$

where $\alpha$ (resp. $\beta$ ) is expressed as the Taylor expansion of $\alpha$ (resp. $\beta$ ) about 1 (resp. -1 ). Let $P_{\ell}, \ell \in \mathbf{N}$ be the Hermite interpolating polynomials to $f$ at $-1,1$ with multiplicities $\ell, \ell$. Then, the following assertions hold:

(1) $f$ has the two point Taylor expansion about $-1,1$ on $(-\sqrt{2}, 0) \cup(0, \sqrt{2})$, that is,

$$
\lim _{\ell \rightarrow \infty} P_{\ell}(x)=f(x) \quad \text { for all } x \in(-\sqrt{2}, 0) \cup(0, \sqrt{2}) \text {. }
$$

(2) Moreover, if $\alpha(0)=\beta(-0)$, then $f$ has the two point Taylor expansion about $-1,1$ on $(-\sqrt{2}, \sqrt{2})$, that is,

$$
\lim _{\ell \rightarrow \infty} P_{\ell}(x)=f(x) \quad \text { for all } x \in(-\sqrt{2}, \sqrt{2}) \text {. }
$$


Theorem 3. (Evaluation of $\left.\lim _{\ell \rightarrow \infty} P_{\ell}(0)\right)$ Let $f$ be a real-valued function on $[-r, r](r>1+\sqrt{2})$ which satisfies the same condition as in Theorem 2 . Let $P_{\ell}, \ell \in \mathbf{N}$ be the Hermite interpolating polynomials to $f$ at $-1,1$ with multiplicities $\ell, \ell$. Then

$$
\lim _{\ell \rightarrow \infty} P_{\ell}(0)=\frac{\alpha(0)+\beta(-0)}{2}
$$

holds.

Theorem 4. (Termwise Differentiation) Let $f$ be a real-valued function on $[-r, r](r>1+\sqrt{2})$ which satisfies the same condition as in Theorem 2 . Let $P_{\ell}, \ell \in \mathbf{N}$ be the Hermite interpolating polynomials to $f$ at $-1,1$ with multiplicities $\ell, \ell$. It holds that, for any given positive integer $k$

$$
\lim _{\ell \rightarrow \infty} P_{\ell}^{(k)}(x)=f^{(k)}(x) \quad \text { for all } x \in(-\sqrt{2}, 0) \cup(0, \sqrt{2}) .
$$

\section{Preliminaries}

First we begin with a proposition which states the existence of Hermite interpolating polynomials.

Proposition 5. (see p. 365 in Kincaid and Cheney [2]) Let $x_{0} \leqq x_{1} \leqq \cdots \leqq$ $x_{n}$ be a list of nodes. In the list of nodes, only distinct nodes $z_{0}, \ldots, z_{p}$ appear and each node $z_{i}, i=0, \ldots, p$ is just appeared $k_{i}$ times. Let $f$ be sufficiently differentiable at $z_{0}, \ldots, z_{p}$. Then, there exists a unique polynomial $p$ of degree at most $n$ satisfying that

$$
p^{(j)}\left(z_{i}\right)=f^{(j)}\left(z_{i}\right), \quad j=0, \ldots, k_{i}-1, \quad i=0, \ldots, p .
$$

In Proposition 5, we call each positive integer $k_{i}, i=0, \ldots, p$ the multiplicity at $x_{i}$. Divided differences of functions can be defined by this proposition.

Definition 2. Let $x_{0} \leqq x_{1} \leqq \cdots \leqq x_{n}$ be a list of nodes and let $f$ be sufficiently differentiable at $x_{0}, \ldots, x_{n}$. Then the coefficient of $x^{n}$ of the polynomial $p$ with the property (2.1) stated above is called the $n$-th order divided difference of $f$ at $x_{0}, \ldots, x_{n}$ and is denoted by $f\left[x_{0}, \ldots, x_{n}\right]$. 
By Definition 2, it is easily seen that the divided difference $f\left[x_{0}\right]$ of a function $f$ at a point $x_{0}$ is equal to $f\left(x_{0}\right)$. The following proposition of a recursive formula and a divided difference table are of much use to calculate divided differences of functions.

Proposition 6. (see p. 372 in Kincaid and Cheney [2]) Let $x_{0} \leqq \cdots \leqq x_{n}$ be a list of nodes and let $f$ be sufficiently differentiable at $x_{0}, \ldots, x_{n}$. Then the divided differences obey this recursive formula:

$$
f\left[x_{0}, \ldots, x_{n}\right]= \begin{cases}\frac{f\left[x_{1}, \ldots, x_{n}\right]-f\left[x_{0}, \ldots, x_{n-1}\right]}{x_{n}-x_{0}} & \left(x_{0} \neq x_{n}\right) \\ \frac{f^{(n)}\left(x_{0}\right)}{n !} & \left(x_{0}=x_{n}\right)\end{cases}
$$

If data points $\left(x_{i}, f\left(x_{i}\right)\right), i=0, \ldots, n$ are given, then we can construct the following divided difference table $T\left[f, x_{0}, \ldots, x_{n}\right]$ from them. By Proposition 6 , the $(i+1)$-th order divided differences in the table are calculated from the $i$-th order divided differences.

$$
\begin{aligned}
& x_{0} \quad f\left[x_{0}\right] \\
& f\left[x_{0}, x_{1}\right] \\
& x_{1} \quad f\left[x_{1}\right] \\
& x_{2} \quad f\left[x_{2}\right] \\
& f\left[x_{1}, x_{2}\right] \\
& \vdots \quad \vdots \quad \vdots \quad \cdots \quad f\left[x_{0}, x_{1}, \cdots, x_{n-1}, x_{n}\right] \\
& x_{n-2} \quad f\left[x_{n-2}\right] \\
& f\left[x_{n-2}, x_{n-1}\right] \\
& x_{n-1} \quad f\left[x_{n-1}\right] \\
& x_{n} \quad f\left[x_{n}\right] \\
& f\left[x_{n-1}, x_{n}\right]
\end{aligned}
$$

Divided difference table $T\left[f, x_{0}, \ldots, x_{n}\right]$

In the divided difference table stated above, we call the column vector consisting of the $i$-th order divided differences the $i$-th order column vector for convenience.

Notation. Let $x_{0} \leqq x_{1} \leqq \cdots \leqq x_{n}$ be a list of nodes and let $f$ be sufficiently differentiable at $x_{0}, \ldots, x_{n}$. In the list of nodes, only distinct points $z_{0}, \ldots, z_{p}$ 
appear and each point $z_{i}, i=0, \ldots, p$ is just appeared $k_{i}$ times. To make sure of multiplicities, we write

$$
f\left[z_{0}, \ldots, z_{p} ; k_{0}, \ldots, k_{p}\right]
$$

for the divided difference $f\left[x_{0}, \ldots, x_{n}\right]$. And the divided difference table $T\left[f, x_{0}\right.$, $\left.\ldots, x_{n}\right]$ is also denoted by $T\left[f ; z_{0}, \ldots, z_{p} ; k_{0}, \ldots, k_{p}\right]$.

Let $x_{0} \leqq x_{1} \leqq \cdots \leqq x_{n}$ be a list of nodes and let $f$ be sufficiently differentiable at $x_{0}, \ldots, x_{n}$. It is well known that the Hermite interpolating polynomial $p$ to $f$ at $x_{0}, \ldots, x_{n}$, which $p$ satisfies (2.1), is expressed as

$$
p(x)=\sum_{j=0}^{n} f\left[x_{0}, \ldots, x_{j}\right] \Pi_{i=0}^{j-1}\left(x-x_{i}\right),
$$

where $\Pi_{i=0}^{-1}\left(x-x_{i}\right)=1$ (see p.370 in Kincaid and Cheney [1]).

The following proposition is a basic statement, but it is a key result to prove our theorems.

Propostion 7. Let $\left(a \leqq x_{0} \leqq x_{1} \leqq \cdots \leqq x_{n}(\leqq b)\right.$ be a list of nodes and let $f$ be a real-valued function on an interval $[a, b]$ which is sufficiently differentiable at $x_{0}, \ldots, x_{n}$. If $p$ is the the Hermite interpolating polynomial to $f$ at $x_{0}, \ldots, x_{n}$, then

$$
f(x)-p(x)=f\left[x, x_{0}, \ldots, x_{n}\right]\left(x-x_{0}\right)\left(x-x_{1}\right) \cdots\left(x-x_{n}\right), \quad x \in[a, b] .
$$

\section{Proofs of Theorems}

We show the following two lemmas before proving Theorem 2 .

Lemma 8. Let $f$ be a real-valued function on $[-r, r](r>1+\sqrt{2})$, which is expressed as

$$
f(x)=\left\{\begin{array}{ll}
\alpha(x) & x \in[0, r] \\
\beta(x) & x \in[-r, 0)
\end{array},\right.
$$

where $\alpha$ (resp. $\beta$ ) is expressed as the Taylor expansion of $\alpha$ (resp. $\beta$ ) about 1 (resp. - 1). Then, the following assertions hold:

(1) $\sup _{i, j \in \mathbf{N} \cup\{0\}, i^{2}+j^{2} \neq 0}|f[-1,1 ; i, j]|<+\infty$

(2) $\sup _{i, j \in \mathrm{N} \cup\{0\}, i^{2}+j^{2} \neq 0}|f[-1, x, 1 ; i, 1, j]|<+\infty \quad$ for each $x \in(-\sqrt{2}, \sqrt{2})$ 
Proof. (1) It is sufficient to show the existence of a positive number $M$ such that

$$
\max \{|b|: b \in T[f ;-1,1 ; n, n]\} \leqq M \text { for all } n \in \mathbf{N} .
$$

Because any $f[-1,1 ; i, j], i, j \in \mathbf{N} \cup\{0\}, i^{2}+j^{2} \neq 0$ belongs to some $T[f ;-1,1 ; n$, $n]$. have

By the condition of $f, \sum_{k=0}^{\infty}\left|\frac{\alpha^{(k)}(1)}{k !}\right|$ and $\sum_{k=0}^{\infty}\left|\frac{\beta^{(k)}(-1)}{k !}\right|$ converge. So we

$$
\begin{gathered}
A=\sup \left\{\left|\frac{\alpha^{(k)}(1)}{k !}\right|: k \in \mathbf{N} \cup\{0\}\right\}<\infty, \\
B=\sup \left\{\left|\frac{\beta^{(k)}(-1)}{k !}\right|: k \in \mathbf{N} \cup\{0\}\right\}<\infty .
\end{gathered}
$$

Since every divided difference in $T[f ;-1,1 ; n, n]$ is a real number expressed as $\frac{\alpha^{(k)}(1)}{k !}, \frac{\beta^{(k)}(1)}{k !}$ or $\frac{b_{1}-b_{2}}{2}$, where $b_{1}, b_{2}$ are divided differences in the same column vector of $T[f ;-1,1 ; n, n]$, if we put $M=\max \{A, B\}$, then from the recursive formula of the divided differences, (3.1) immediately follows.

(2) In case $x=1$ or -1 , the assertion (2) is reduced to the assertion (1). Hence, without loss of generality, we assume that $0 \leq x<\sqrt{2}$ and $x \neq 1$.

Let $x$ be a given nonnegative number which is less than $\sqrt{2}$. As in the analogous way to the proof of (1), it is sufficient to show the existence of a postive number $L$ such that

$$
\max \{|b|: b \in T[f ;-1, x, 1 ; n, 1, n]\} \leqq L \text { for all } n \in \mathbf{N} .
$$

Because any $f[-1, x, 1 ; i, 1, j]$ belongs to some $T[f ;-1, x, 1 ; n, 1, n]$. The 0 -th and the first column vectors of $T[f ;-1, x, 1 ; n, 1, n]$ are as follows: 


$$
\begin{array}{cc}
f(-1)=\beta(-1) & \frac{\beta^{\prime}(-1)}{1 !} \\
f(-1)=\beta(-1) & \vdots \\
\vdots & \frac{\beta^{\prime}(-1)}{1 !} \\
f(-1)=\beta(-1) & \frac{\alpha(x)-\beta(-1)}{x+1} \\
f(x)=\alpha(x) & \frac{\alpha(x)-\alpha(1)}{x-1} \\
f(1)=\alpha(1) & \frac{\alpha^{\prime}(1)}{1 !} \\
f(1)=\alpha(1) & \frac{\alpha^{\prime}(1)}{1 !} \\
f(1)=\alpha(1) & \vdots \\
\vdots &
\end{array}
$$

The $k(2 \leq k \leq n-1)$-th column vector of the divided difference table is as follows: 


$$
\begin{gathered}
\frac{\beta^{(k)}(-1)}{k !} \\
\frac{\beta^{(k)}(-1)}{k !} \\
\vdots \\
\frac{\beta^{(k)}(-1)}{k !} \\
\frac{1}{\left(x+\frac{1}{k}\left\{\alpha(x)-\left(\beta(-1)+\frac{\beta^{\prime}(-1)}{1 !}(x+1)+\cdots+\frac{\beta^{(k-1)}(-1)}{(k-1) !}(x+1)^{k-1}\right)\right\}\right.} \\
\frac{1}{2}\{f[-1, x, 1 ; k-2,1,1]-f[-1, x ; k-1,1]\}=f[-1, x, 1 ; k-1,1,1] \\
\frac{1}{2}\{f[-1, x, 1 ; k-3,1,2]-f[-1, x, 1 ; k-2,1,1]\}=f[-1, x, 1 ; k-2,1,2] \\
\vdots \\
\frac{1}{2}\{f[x, 1 ; 1, k-1]-f[-1, x, 1 ; 1,1, k-2]\}=f[-1, x, 1 ; 1,1, k-1] \\
\left.\left.\frac{\alpha^{\prime}(1)}{1 !}(x-1)+\cdots+\frac{\alpha^{(k-1)}(1)}{(k-1) !}(x-1)^{k-1}\right)\right\} \\
\frac{\alpha^{(k)}(1)}{k !} \\
\vdots \alpha(x)-(\alpha(1)+
\end{gathered}
$$

And the $n$-th and the $n+1$-th column vectors of the divided difference table is as follows: 


$$
\begin{gathered}
\frac{1}{(x+1)^{n}}\left\{\alpha(x)-\left(\beta(-1)+\frac{\beta^{\prime}(-1)}{1 !}(x+1)+\cdots+\frac{\beta^{(n-1)}(-1)}{(n-1) !}(x+1)^{n-1}\right)\right\} \\
\frac{1}{2}\{f[-1, x, 1 ; n-2,1,1]-f[-1, x ; n-1,1]\}=f[-1, x, 1 ; n-1,1,1] \\
\frac{1}{2}\{f[-1, x, 1 ; n-3,1,2]-f[-1, x, 1 ; n-2,1,1]\}=f[-1, x, 1 ; n-2,1,2] \\
\vdots \\
\frac{1}{2}\{f[x, 1 ; 1, n-1]-f[-1, x, 1 ; 1,1, n-2]\}=f[-1, x, 1 ; 1,1, n-1] \\
\frac{1}{(x-1)^{n}}\left\{\alpha(x)-\left(\alpha(1)+\frac{\alpha^{\prime}(1)}{1 !}(x-1)+\cdots+\frac{\alpha^{(n-1)}(1)}{(n-1) !}(x-1)^{n-1}\right)\right\}
\end{gathered}
$$

and

$$
\begin{gathered}
\frac{1}{2}\{f[-1, x, 1 ; n-1,1,1]-f[-1, x ; n, 1]\}=f[-1, x, 1 ; n, 1,1] \\
\frac{1}{2}\{f[-1, x, 1 ; n-2,1,2]-f[-1, x, 1 ; n-1,1,1]\}=f[-1, x, 1 ; n-1,1,2] \\
\vdots \\
\frac{1}{2}\{f[x, 1 ; 1, n]-f[-1, x, 1 ; 1,1, n-1]\}=f[-1, x, 1 ; 1,1, n]
\end{gathered}
$$

We see that every divided difference in $T[f ;-1, x, 1 ; n, 1, n]$ is a real number expressed as one of the following four types:

(a) $A_{k}=\frac{\alpha^{(k)}(1)}{k !}, B_{k}=\frac{\beta^{(k)}(-1)}{k !}$

(b) $C_{k}=\frac{1}{(x+1)^{k}}\left\{\alpha(x)-\left(\beta(-1)+\frac{\beta^{\prime}(-1)}{1 !}(x+1)+\cdots+\right.\right.$

$$
\left.\left.\frac{\beta^{(k-1)}(-1)}{(k-1) !}(x+1)^{k-1}\right)\right\}
$$

(c) $D_{k}=\frac{1}{(x-1)^{k}}\left\{\alpha(x)-\left(\alpha(1)+\frac{\alpha^{\prime}(1)}{1 !}(x-1)+\cdots+\right.\right.$

$$
\left.\left.\frac{\alpha^{(k-1)}(1)}{(k-1) !}(x-1)^{k-1}\right)\right\}
$$


(d) $\frac{d_{1}-d_{2}}{2}$, where $d_{1}, d_{2}$ are divided differences in the same column vector of $T[f ;-1, x, 1 ; n, 1, n]$

Hence, if we put $A=\sup \left\{\left|A_{k}\right|: k \in \mathbf{N} \cup\{0\}\right\}, B=\sup \left\{\left|B_{k}\right|: k \in \mathbf{N} \cup\{0\}\right\}$, $C=\sup \left\{\left|C_{k}\right|: k \in \mathbf{N}\right\}, D=\sup \left\{\left|D_{k}\right|: k \in \mathbf{N}\right\}$ and $L=\max \{A, B, C, D\}$, then from the condition of $f, L$ is finite. Moreover, from the recursive formula of the divided differences, (3.2) immediately follows.

Lemma 9. Let $f$ be a real-valued function on $[-r, r](r>1+\sqrt{2})$, which is expressed as

$$
f(x)=\left\{\begin{array}{ll}
\alpha(x) & x \in[0, r] \\
\beta(x) & x \in[-r, 0)
\end{array},\right.
$$

where $\alpha$ (resp. $\beta$ ) is expressed as the Taylor expansion of $\alpha$ (resp. $\beta$ ) about 1 (resp. -1$)$. Suppose that $\alpha(0)=\beta(-0)$. Then, it holds that

$$
\lim _{\ell \rightarrow \infty} f[-1,0,1 ; \ell, 1, \ell]=0 .
$$

Proof. We will show that for any given positive number $\varepsilon$, there exists a positive integer $\ell_{0}$ such that $|f[-1,0,1 ; \ell, 1, \ell]|<2 \varepsilon$ for all $\ell \geq \ell_{0}$.

Suppose that by Lemma $8, M$ is a positive number with

$$
\sup \left\{f[-1,0,1 ; i, 1, j]: i, j \in \mathbf{N} \cup\{0\}, i^{2}+j^{2} \neq 0\right\} \leq M .
$$

From the proof of Lemma 8 , we can find a positive integer $m(\geqq 2)$ satisfying

$$
\max \left\{\left|A_{k}\right|,\left|B_{k}\right|,\left|C_{k}\right|,\left|D_{k}\right|\right\}<\varepsilon
$$

for all $k \geq m$, where $A_{k}, B_{k}, C_{k}, D_{k}$ denote the numbers in the proof of Lemma 8.

Assume that a positive integer $\ell$ is much larger than $m$. Then, from (3.3) and (3.4), we observe that the $m$-th column vector $\mathbf{a}^{(m)}=\left(a_{i}^{(m)}\right)_{1 \leq i \leq 2 \ell+1-m} \in$ $\mathbf{R}^{2 \ell+1-m}$ of $T[f ;-1,0,1 ; \ell, 1, \ell]$ satisfies

$$
\begin{aligned}
& \left|a_{i}^{(m)}\right|<\varepsilon, \quad 1 \leq i \leq \ell-m+1, \quad \ell+1 \leq i \leq 2 \ell+1-m \\
& \left|a_{i}^{(m)}\right| \leq M, \quad \ell-m+2 \leq i \leq \ell .
\end{aligned}
$$

On the other hand, we introduce a recursive relation by which a column vector $\mathbf{c}^{\prime}=\left(c_{i}^{\prime}\right)_{1 \leq i \leq n}$ is obtained from a column vector $\mathbf{c}=\left(c_{i}\right)_{1 \leq i \leq n+1}$ such that

$$
c_{i}^{\prime}=\frac{c_{i}+c_{i+1}}{2}, \quad i=1,2, \ldots, n .
$$


Let $\mathbf{c}^{(1)} \in \mathbf{R}^{r}$ be an initial column vector and let $\mathbf{c}^{(k)}, 2 \leq k \leq n$ be the $k$-th column vector which is obtained by the recursive relation (3.6). It is easily seen that for an initial column vector $\mathbf{c}^{(1)}=\left(c_{i}^{(1)}\right)_{1 \leq i \leq 2 \ell+1-m} \in \mathbf{R}^{2 \ell+1-m}$ with

$$
c_{i}^{(1)}= \begin{cases}\varepsilon \quad, \quad 1 \leq i \leq \ell-m+1, \quad \ell+1 \leq i \leq 2 \ell+1-m \\ 0 \quad, \quad \ell-m+2 \leq i \leq \ell .\end{cases}
$$

As an estimation of the absolute value of $\mathbf{c}^{(2 \ell+1-m)} \in \mathbf{R}$ obtained by (3.6), we have

$$
\left|\mathbf{c}^{(2 \ell+1-m)}\right|<\varepsilon
$$

Let us consider another initial column vector $\mathbf{d}^{(1)}=\left(d_{i}^{(1)}\right)_{1 \leq i \leq 2 \ell+1-m} \in$ $\mathbf{R}^{2 \ell+1-m}$ with

$$
d_{i}^{(1)}=\left\{\begin{array}{cl}
0 & , \quad 1 \leq i \leq \ell-m+1, \quad \ell+1 \leq i \leq 2 \ell+1-m \\
M & , \quad \ell-m+2 \leq i \leq \ell
\end{array}\right.
$$

By (3.5), we have $a b s\left(\mathbf{a}^{(m)}\right) \leq \mathbf{c}^{(\mathbf{1})}+\mathbf{d}^{(\mathbf{1})}$, which means that $\left|a_{i}^{(m)}\right| \leq$ $c_{i}^{(1)}+d_{i}^{(1)}, i=1, \ldots, 2 \ell+1-m$. Futhermore, from the recursive relations of divided differences and (3.6), we obtain

$$
a b s\left(\mathbf{a}^{(m+k)}\right) \leq \mathbf{c}^{(1+k)}+\mathbf{d}^{(1+k)}, \quad 0 \leq k \leq 2 \ell-m
$$

and in particular,

$$
\left|\mathbf{a}^{(2 \ell)}\right| \leq \mathbf{c}^{(2 \ell+1-m)}+\mathbf{d}^{(2 \ell+1-m)} .
$$

Moreover, by the proof of Theorem in Kitahara, Chiyonobu and Tsukamoto [3], there exists a positive integer $p$ such that $0 \leq \mathbf{d}^{(2 \ell+1-m)}<\varepsilon$ for all $\ell \geq p$. So, if we put $\ell_{0}=\max \{m, p\}$, then from $(3.7)$ and $(3,8)$, it follows that $\left|\mathbf{a}^{(2 \ell)}\right|=|f[-1,0,1 ; \ell, 1, \ell]|<2 \varepsilon$ for all $\ell \geq \ell_{0}$. This completes the proof.

Now we are in position to prove Theorem 2.

Proof of Theorem 2. For each positive integer $\ell$, let $P_{\ell}$ be the Hermite interpolating polynomial to $f$ at $-\ell, \ell$ with multiplicities $\ell, \ell$. By Proposition 7 , we have for each $x \in(-\sqrt{2}, \sqrt{2})$

$$
\begin{aligned}
\left|f(x)-p_{\ell}(x)\right|=\mid(x-1)^{\ell}(x+1)^{\ell} f[-1, x & , 1 ; \ell, 1, \ell] \mid \\
& =\left|x^{2}-1\right|^{\ell}|f[-1, x, 1 ; \ell, 1, \ell]| .
\end{aligned}
$$

(1) Since $\left|x^{2}-1\right|<1$ for each $x \in(-\sqrt{2}, 0) \cup(0,-\sqrt{2})$, it is sufficient to show

$$
\sup _{\ell \geqq 1}|f[-1, x, 1 ; \ell, 1, \ell]|<+\infty \text {. }
$$




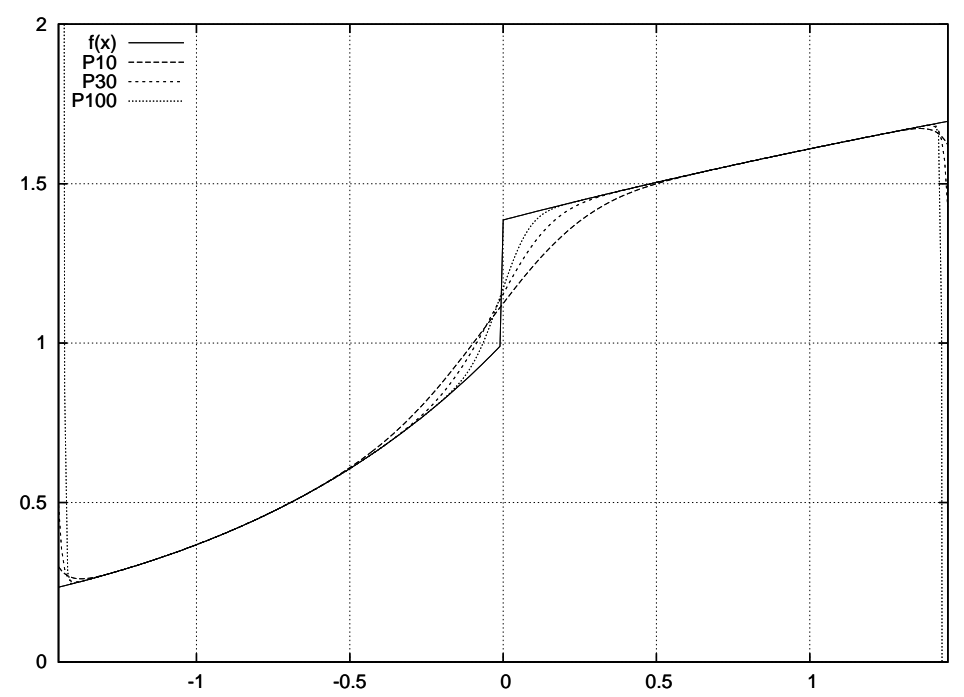

But this immediately follows from Lemma 8 (2).

(2) Without loss of generality, we assumu that $\alpha(0)=\beta(-0)=0$. From (3.9), we have

$$
\left|f(0)-P_{\ell}(0)\right|=\left|(0-1)^{\ell}(0+1)^{\ell} f[-1,0,1 ; \ell, 1, \ell]\right|=|f[-1,0,1 ; \ell, 1, \ell]| .
$$

By Lemma 9, since $\lim _{n \rightarrow \infty} f[-1,0,1 ; \ell, 1, \ell]=0$, we get $\lim _{n \rightarrow \infty} P_{\ell}(0)=$ $f(0)$. From this and (1), it holds that $\lim _{n \rightarrow \infty} P_{\ell}(x)=f(x)$ for all $x \in(-\sqrt{2}$, $-\sqrt{2})$.

Example. As a function on $[-3,3]$ which satisfies the condition of Theorem 2 , let us consider $f(x)=\left\{\begin{array}{ll}\log (4+x) & x \in[0,3] \\ e^{x} & x \in[-3,0) .\end{array}\right.$ Let $P \ell$ denote the Hermite interpolating polynomials to $f$ at $-1,1$ with multiplicities $\ell, \ell$ for $\ell=10,30,100$, respectively. One can see that as multiplicities get larger, the Hermite interpolating polynomials get closer to $f(x)$ on $(-1.4,1.4)$.

We prepare the following lemma to prove Theorem 3.

Lemma 10. Let $f$ be a real-valued function on $\mathbf{R}$ which is expressed as

$$
f(x)= \begin{cases}1 & x \in(0, \infty) \\ 0 & x=0 \\ -1 & x \in(-\infty, 0)\end{cases}
$$


Let $P_{\ell}, \ell \in \mathbf{N}$ be the Hermite interpolating polynomials to $f$ at $-1,1$ with multiplicities $\ell, \ell$. Then, it holds that $P_{\ell}(0)=0, \ell \in \mathbf{N}$.

Proof. By Proposition 7, we have for each $x \in \mathbf{R}$

$$
f(x)-P_{\ell}(x)=(x-1)^{\ell}(x+1)^{\ell} f[-1, x, 1 ; \ell, 1, \ell] .
$$

Since $f(0)-P_{\ell}(0)=(-1)^{\ell} f[-1,0,1 ; \ell, 1, \ell]$, it is sufficient to show $f[-1, x, 1$; $\ell, 1, \ell]=0, \ell \in \mathbf{N}$. The tranpose of the 0 -th and the 1 -st column vectors of $T[f$ : $-1,0,1 ; \ell, 1, \ell]$ are $-1, \ldots,-1,0,1, \ldots, 1$ and $0, \ldots, 0,1,1,0, \ldots, 0$, respectively. Hence, we guess that the transpose of any even numbered colomn vector is expressed as

$$
-a_{m},-a_{m-1}, \ldots,-a_{1}, 0, a_{1}, \ldots, a_{m-1}, a_{m}
$$

and the transpose of any odd numbered colum vector is expressed as

$$
b_{m}, b_{m-1}, \ldots, b_{1}, b_{1}, \ldots b_{m-1}, b_{m}
$$

Clearly, (3.10), (3.11) hold for the 0-th and the 1-st column vector. If an even (odd) numbered column vector is expressed as $(3.10)((3.11))$, we easily see that the next odd (even) numbered colomn vector is expressed as $(3.11)((3.10))$. Hence, any column vector of $T[-1,0,1 ; \ell, 1, \ell]$ is expressed as (3.10) or (3.11).

Consequently, we obtain the $2 \ell$-th column vector $f[-1,0,1 ; \ell, 1, \ell]=0$.

Corollary 11. Let $f$ be a real-valued function on $\mathbf{R}$ which is expressed as

$$
f(x)= \begin{cases}C_{1} & x \in[0, \infty) \\ C_{2} & x \in(-\infty, 0),\end{cases}
$$

where $C_{1}$ and $C_{2}$ are real numbers. Let $P_{\ell}, \ell \in \mathbf{N}$ be the Hermite interpolating polynomials to $f$ of $-1,1$ with multiplicities $\ell, \ell$. Then, it holds that $P_{\ell}(0)=$ $\frac{C_{1}+C_{2}}{2}, \ell \in \mathbf{N}$.

Now we show a proof of Theorem 3 .

Proof of Theorem 3. First we consider two functions $g(x), h(x)$ such that

$$
g(x)= \begin{cases}\alpha(x) & x \in[0, \sqrt{2}) \\ \beta(x)-\beta(-0)+\alpha(0) & x \in(-\sqrt{2}, 0) .\end{cases}
$$

and

$$
h(x)= \begin{cases}0 & x \in[0, \sqrt{2}) \\ \beta(-0)-\alpha(0) & x \in(-\sqrt{2}, 0) .\end{cases}
$$


$g(x), h(x)$ are real-valued functions on $(-\sqrt{2}, \sqrt{2})$ which satisfy the condition of Theorem 2 and $g(x)$ is continuous on $(-\sqrt{2}, \sqrt{2})$. Let $P_{\ell}, Q_{\ell}, R_{\ell}, \ell \in \mathbf{N}$ be the Hermite interpolating polynomials to $f, g, h$ at $-1,1$ with multiplicities $\ell, \ell$, respectively. Since $f(x)=g(x)+h(x), x \in(-\sqrt{2}, \sqrt{2})$, by Theorem $2(2)$ and Corollary 11, we have

$$
\lim _{\ell \rightarrow \infty} P_{\ell}(0)=\lim _{\ell \rightarrow \infty}\left(Q_{\ell}(0)+R_{\ell}(0)\right)=\alpha(0)+\frac{\beta(-0)-\alpha(0)}{2}=\frac{\alpha(0)+\beta(-0)}{2} .
$$

Finally, we turn to prove Theorem 4 .

Proof of Theorem 4. Let $x$ be any given number in $(-\sqrt{2}, 0) \cup(0, \sqrt{2})$ and let $k$ be any given positive integer. Suppose that a positive integer $\ell$ is lager than $k$. For a given real-valued function $f$ on $[-r, r](r>1+\sqrt{2})$ which satisfies the same condition as in Theorem 2, we put the Hermite interpolating polynomials $P_{\ell}$ to $f$ at $-1,1$ with multiplicities $\ell, \ell$

$$
\begin{array}{r}
P_{\ell}(x)=f[-1,1 ; \ell, \ell](x+1)^{\ell}(x-1)^{\ell-1}+f[-1,1 ; \ell, \ell-1](x+1)^{\ell-1}(x-1)^{\ell-1}+\cdots \\
+f[-1,1 ; 1,0]=U_{2 \ell-1}(x)+U_{2 \ell-2}(x)+\cdots+U_{0}(x), \quad(3.12)
\end{array}
$$

where $U_{2 k-1}(x)=f[-1,1 ; k, k](x+1)^{k}(x-1)^{k-1}$ and $U_{2 k-2}(x)=f[-1,1 ; k, k-$ $1](x+1)^{k-1}(x-1)^{k-1}, 1 \leq k \leq \ell$. By Lemma 8 (1), we put

$$
M=\sup _{i, j \in \mathbf{N} \cup\{0\}, i^{2}+j^{2} \neq 0}|f[-1,1 ; i, j]| .
$$

Let $R_{\ell-k}$ be the Hermite interpolating polynomials to $f^{(k)}$ at $-1,1$ with multiplicities $\ell-k, \ell-k$. Since $R_{\ell-k}$ and the $k$-th order derivative $P_{\ell}^{(k)}$ of $P_{\ell}$ satisfy

$$
\left(P_{\ell}^{(k)}\right)^{(i)}( \pm 1)=\left(R_{\ell-k}\right)^{(i)}( \pm 1)=f^{(k+i)}( \pm 1), \quad 0 \leq i \leq \ell-k-1,
$$

we have

$$
\begin{aligned}
P_{\ell}^{(k)}(x)-R_{\ell-k}(x)= & (x+1)^{\ell-k}(x-1)^{\ell-k}\left(a_{k-1, \ell} x^{k-1}+a_{k-2, \ell} x^{k-2}+\cdots+a_{0, \ell}\right) \\
& =c_{2 \ell-k-1} x^{2 \ell-k-1}+\cdots+c_{2 \ell-2 k} x^{2 \ell-2 k}+\cdots+c_{0} . \quad(3.13)
\end{aligned}
$$

Then, it is sufficient to show the existence of a polynomial $S(\ell)$ with

$$
\left|a_{k-1, \ell} x^{k-1}+a_{k-2, \ell} x^{k-2}+\cdots+a_{0, \ell}\right|<S(\ell), x \in(-\sqrt{2}, 0) \cup(0, \sqrt{2}) .
$$

Because, if (3.14) holds, since $\left|x^{2}-1\right|<1$,

$$
\lim _{\ell \rightarrow \infty}\left|P_{\ell}^{(k)}(x)-R_{\ell-k}(x)\right|=\lim _{\ell \rightarrow \infty}\left|x^{2}-1\right|^{\ell-k} S(\ell)=0 .
$$


We put $(x+1)^{\ell-k}(x-1)^{\ell-k}=x^{2 \ell-2 k}+b_{2 \ell-2 k-1} x^{2 \ell-2 k-1}+\cdots+b_{2 \ell-3 k+1} x^{2 \ell-3 k+1}+\cdots+b_{0}$.

Since the absolute value of each coefficient $b_{i}, 2 \ell-3 k+1 \leq i \leq 2 \ell-2 k-1$, is smaller than the coefficient of $x^{2 \ell-k}$ of $(x+1)^{2 \ell}$, we have

$$
\left|b_{i}\right|<\left(\begin{array}{c}
2 \ell \\
k
\end{array}\right)<(2 \ell)^{k} .
$$

Furthermore, each $c_{2 \ell-2 k+t}, 0 \leq t \leq k-1$ satisfies

$$
c_{2 \ell-2 k+t}=a_{t, \ell}+a_{t+1, \ell} b_{2 \ell-2 k-1}+\cdots+a_{k-1, \ell} b_{2 \ell-3 k+t+1} .
$$

Noting that $R_{\ell-k}(x)$ is a polynomial of degree at most $2 \ell-2 k-1$, from (3.12) and (3.13), we obtain, for $0 \leq t \leq k-1$

$\left|c_{2 \ell-2 k+t}\right| \leq\left(\left(\right.\right.$ the absolute value of the coefficient of $x^{2 \ell-k+t}$ of $\left.U_{2 \ell-1}\right)$

$+\cdots+\left(\right.$ the absolute value of the coefficient of $x^{2 \ell-k+t}$

$$
\text { of } \left.\left.U_{2 \ell-k+t}\right)\right)(2 \ell-k+t) \cdots(2 \ell-2 k+t+1)
$$

$$
\begin{array}{r}
\leq M\left(\begin{array}{c}
2 \ell \\
k
\end{array}\right)(k-t)(2 \ell-k+1) \cdots(2 \ell-2 k+t+1)<M(2 \ell)^{k} k(2 \ell)^{k}=4^{k} k M \ell^{2 k} \\
=L \cdot \ell^{2 k}, \quad(3.17)
\end{array}
$$

where $L=4^{k} k M$.

Now we will give estimations of the absolute values of $a_{k-1, \ell}, \ldots, a_{0, \ell}$. For $\left|a_{k-1, \ell}\right|$, by (3.16) and (3.17) we have

$$
\left|a_{k-1, \ell}\right|=\left|c_{2 \ell-k-1}\right|<L \ell^{2 k}=S_{1}(\ell) .
$$

$S_{1}(\ell)$ is a polynomial of degree $2 k$. For $\left|a_{k-2, \ell}\right|$, since, by (3.16) and (3.17),

$$
\left|c_{2 \ell-k-2}\right|=\left|a_{k-2, \ell}+a_{k-1, \ell} b_{2 \ell-2 k-1}\right|<L \ell^{2 k},
$$

by the triangle inequality and (3.15), we get

$$
\left|a_{k-2, \ell}\right|<L \ell^{2 k}+S_{1}(\ell)(2 \ell)^{k}=S_{2}(\ell) .
$$

$S_{2}(\ell)$ is a polynomial of degree $3 k$. Repeating this procedure, we see that

$$
\left|a_{k-i, \ell}\right|<S_{i}(\ell), \quad 1 \leq i \leq k,
$$


where each $S_{i}(\ell)$ is a polynomial of degree $(i+1) k$. Hence, if we put $S(\ell)=$ $2^{k}\left(S_{1}(\ell)+S_{2}(\ell)+\cdots+S_{k}(\ell)\right)$, then $S(\ell)$ is a polynomial of degree $k(k+1)$ and we easily have

$$
\begin{array}{r}
\left|a_{k-1, \ell} x^{k-1}+a_{k-2, \ell} x^{k-2}+\cdots+\right| a_{0, \ell} \mid \leq 2^{k}\left(\left|a_{k-1, \ell}\right|+\left|a_{k-2, \ell}\right|+\cdots+\left|a_{0, \ell}\right|\right) \\
<2^{k}\left(S_{1}(\ell)+S_{2}(\ell)+\cdots+S_{k}(\ell)\right)=S(\ell) .
\end{array}
$$

This completes the proof.

In this note, we show a second step to the problem "What functions does $\mathcal{T}_{m}[I]$ consist of ?" Finally, we give problems which lead to a third step to this problem.

Problems. (1) Consider cases corresponding to Theorem 2 of $m(\geq 3)$ point Taylor expansions.

(2) Find other types of functions which belong to $\mathcal{T}_{m}[I](m \geq 2)$.

\section{Acknowledgments}

The first author would like to express to Prof. M. Jimichi his gratitude for his valuable comments on this article.

This research was partially supported by Grant-in-Aid for Scientific Research, Ministry of Education, Culture, Sports, Science and Technology, Japanese Government, No. 22540162.

\section{References}

[1] P.J. Davis, Interpolation \& Approximation (Dover Edition), Dover, New York (1974).

[2] D. Kincaid, W. Cheney, Numerical Analysis, 2-nd Edition, Brooks/Cole Publishing Company, New York (1996).

[3] K. Kitahara, T. Chiyonobu, H. Tsukamoto, A Note on two point Taylor expansion, International Journal of Pure and Applied Mathematics, $\mathbf{7 5}$ (2012), 327-338.

[4] J.L. López, N.M. Temme, Two-point Taylor expansion of analytic functions, Studies in Applied Mathematics, 109 (2002), 297-311, doi: 10.1111/1467-9590.00225. 
[5] J.L. López, N.M. Temme, Multi-point Taylor expansions of analytic functions, Trans. Am. Math. Soc., 356 (2004), 4323-4342.

[6] J.L. Walsh, Interpolation and Approximation by Rational Functions in the Complex Domain, American Mathematical Society, Providence (1969). 